\title{
A Value-based Framework for Internet Peering Agreements
}

\author{
Amogh Dhamdhere \\ CAIDA \\ Constantine Dovrolis \\ Georgia Tech
}

\author{
Pierre Francois \\ Université catholique de Louvain
}

\begin{abstract}
Internet Service Providers (ISPs) use complex peering policies, stipulating various rules for peering with other networks. Peering strategy is often considered a "black art" rather than science, and the outcome of a peering negotiation can depend on factors that are neither technical nor economic. Consequently, ISPs are required to make difficult decisions about the set of networks they should peer with, and the price they should demand/offer to ensure a stable peering link. We propose a quantitative framework for settlement-free and paid-peering links, based on the "value" of a peering link, i.e., the benefit that networks see from that link. We first study a solution where a centralized oracle determines a provably stable, optimal and fair price for a paid-peering link, based on perfect knowledge of the revenues and costs of each network. We next show that with perfect knowledge, the centralized solution can be implemented individually by the peering networks. We then study the effects of inaccurate estimation of peering value by the peering networks. Finally, we examine how value-based peering affects the density of peering links, the nature of end-to-end paths, and the profitability of various network types in the global Internet.
\end{abstract}

\section{INTRODUCTION}

Peering by Internet Service Providers (ISPs) is widely considered to be an art rather than science. With settlement-free peering, two networks allow access to each other's customers for free, and the only cost is that of maintaining the peering link. The decision that networks must make is whether to form a peering link, and periodically, whether to keep that link. A different form of peering called "paid peering" involves routing decisions that are similar to settlement-free peering, but one network pays the other. In such a relationship, the decision is not only about whether to peer, but also which network should pay the other, and how much. Peering coordinators are thus faced with difficult questions about which networks to peer with and how much money to offer/demand, based strictly on knowledge of their own network and limited information about the neighbor network. They must take their decision with limited ability to forecast the possible future implications of their peering decisions. To deal with this problem, several "rules of thumb" peering guidelines have been employed by network operators, such as "tier-1 providers should engage in restrictive peering" or "networks should peer only if traffic ratios are balanced" (discussed in Section II). There are several unanswered questions here. Do these rules lead to peering links that should not exist, or preclude the formation of peering links that should? Are the resulting peering links stable, or does one peer have the incentive to unilaterally depeer the other, possibly causing global unreachability events? What is missing is a quantitative framework for ISPs to make decisions about their peers and the amount of money they should offer/demand.

In this paper, we present a quantitative framework for the creation and evaluation of settlement-free and paid-peering relationships. Our framework is based on the "value" of a peering link for the participating networks. This value represents the monetary benefit from the peering link, i.e., the amount of money a network saves, that it would otherwise pay to its transit providers. We first assume that a centralized oracle proposes a paid-peering price, using complete knowledge of the costs incurred by each network. This price is fair (both networks see the same value from the peering link after this price is paid), optimal (peering at this price is the best possible option for both networks), and stable (no network has the incentive to unilaterally depeer the other), as long as the aggregate value of the peering link for the two networks is positive. We contrast this with cost-benefit peering, where both networks must see a positive value from the peering link. We also show how each network can, with perfect knowledge, individually determine the same price as the oracle.

Next, we discuss practical issues faced by networks in estimating the value of a peering link. We present mechanisms by which a network can accurately measure its own value from the peering link. We also show that a network can only approximately estimate the value of the peering link for the other network. We find that overestimation of the value of a peering link by one or both networks results in a situation where the peering link may not be formed. This represents a common real-world situation where both networks believe that the other gains larger utility from the peering link, and hence cannot find a mutually agreeable price for peering. We find that underestimation of the value of a peering link allows the peering link to be formed, but the payment is higher or lower than the fair peering price. We find that it is beneficial for a network to "hide" its own value, but only if it is able to accurately estimate the value for the other network.

Finally, we use ITER [1] to study the impact of valuebased peering on the density of peering links, end-to-end traffic flow, and profitability of different types of networks in the global Internet. We find that value-based peering can result in a much higher density of peering links, leading to shorter endto-end paths than those seen with traffic-ratio or cost-benefit based peering. We also find that certain networks participate in peering relationships where they pay one network, but get paid by another, i.e, the same network can be both the payer 
and the payee in different paid-peering relationship.

\section{WHAT'S HAPPENING IN THE REAL WORLD}

We present an overview of peering practices commonly used by ISPs, and try to provide the intuition underlying these rules. The "applicant" is the ISP that wishes to peer with another ISP. The "target" is the ISP that the applicant requests for peering.

We were able to classify settlement-free peering criteria into various categories, based on what the target requires from the applicant. We created these categories using peering policies published online by large transit providers, e.g., AOL, AT\&T, Verizon Business, Qwest and British Telecom and regional providers such as Free, Neuf Telecom and Comcast ${ }^{1}$. Some peering rules specify a minimum performance level (e.g., capacities of backbone links) that the applicant must guarantee. We found peering rules related to the geographical spread of the applicant network, specifying a minimum number of POPs that the applicant must have. Some rules specify that the applicant must peer with the target network at a given minimum number of locations. The widely used "traffic ratio" rule specifies that the applicant and the target must exchange at least a minimum amount of traffic over the peering link, with an inbound:outbound traffic ratio at the target lower than a threshold (typically from 1.2 to 2 ). A category of peering requirements relates to traffic routed between the peering networks, e.g., the applicant must have an AS number and must advertise at least a certain maximum prefix size. Some peering rules specify that the applicant will be "evaluated" every few months to verify that it continues to meet the peering criteria. Applicants that do not meet the criteria are given a deadline to comply, failing which they may be offered paidpeering or depeered. Finally, there are non-technical criteria, e.g., the applicant must have a Network Operations Center that operates $24 \times 7$, and sign a Non-disclosure agreement (NDA).

Networks typically set such peering rules to restrict their set of potential peers, in an attempt to peer with the "right" set of peers. This is evident in the rules that only allow networks of similar size, geographical spread, or backbone capacity to peer with the target ("restrictive" peering). Rules that deal with the traffic exchanged over the peering link are from a costbenefit perspective. If the amount of traffic exchanged over the peering link is too small, it does not justify the costs of setting up the peering link. The traffic ratio rule attempts to achieve "fairness" in the peering relationship, especially in the presence of hot-potato routing. With hot potato routing, a large inbound:outbound traffic ratio at the target means that it carries larger traffic volumes for potentially larger distances.

These peering rules appear ad-hoc and arbitrary; it is not clear whether they have general applicability for the target. Do these rules ensure that the target always peers with the "right" set of networks? Further, the aforementioned rules are for settlement-free peering, and don't allow a relationship where routing is as in settlement-free peering, but one network

\footnotetext{
${ }^{1}$ See, for example, http://www.corp.att.com/peering/ (AT\&T), http://www. atdn.net/peering.shtml (AOL), http://www.verizonbusiness.com/terms/peering/ (Verizon), http://www.qwest.com/legal/peering_na.html (Qwest).
}

pays the other. In subsequent sections, we build a quantitative framework that can be applied to both settlement-free and paid-peering, and enable ISPs to make rational decisions about specific peering relationships.

\section{A VALUE-BASED PEERING FRAMEWORK}

In this section, we consider the situation where two networks A and B must decide whether to form a peering link, and if so, the price that one network should pay the other. The price that is set must satisfy the following criteria:

1. The paid peering price must be fair for each network.

2. Forming the peering link with the associated price must be the optimal decision for both $\mathrm{A}$ and $\mathrm{B}$.

3. The paid-peering link must be stable; neither A nor B should benefit by unilaterally depeering the other.

We start with an idealized case where a central entity that has accurate knowledge of the costs incurred by $\mathrm{A}$ and $\mathrm{B}$ can determine a price that is fair, optimal and leads to a stable peering link. We then show how A and B can reach exactly the same solution individually, in the case where they have perfect knowledge of each other's costs. Finally, we consider the case where $\mathrm{A}$ and $\mathrm{B}$ may not be able to accurately determine the costs of the other network.

\section{A. Price setting by a centralized oracle}

We first consider a centralized entity that must determine a compensation price $P$, the amount $\mathrm{B}$ pays to $\mathrm{A}$ to maintain the peering link. If $P$ is positive, $B$ pays $P$ to $A$, otherwise $A$ pays $-P$ to $B$. Settlement-free peering is a special case where $P=0$. The peering link is maintained only if both parties agree on the compensation price, else it is removed.

Let $f_{A}^{n}$ denote the "fitness" of network $\mathrm{A}$, when the peering link is not present. Fitness is the revenue of A (e.g., from customers) minus the costs that $\mathrm{A}$ incurs to manage its network and handle traffic. Both revenues and costs of A can change if the peering link is formed (discussed in Section IV). Let $f_{A}^{p}$ denote the fitness when A peers with B. Let $f_{B}^{n}$ and $f_{B}^{p}$ denote the corresponding values for $\mathrm{B}$. We first assume that the central oracle knows these four quantities accurately. The "value" of the peering link to $\mathrm{A}$ is $V_{A}=f_{A}^{p}-f_{A}^{n}$ and $V_{B}=f_{B}^{p}-f_{B}^{n}$.

1) "Fair" price setting by the oracle: The oracle must determine a price $P$ that meets the fairness criterion, which means that both A and B should see the same benefit from the peering link - the difference in the fitness with and without the peering link should be the same for both A and B. When the peering link is formed with a compensation price $P, \mathrm{~A}$ receives a payment of $P$ from $\mathrm{B}$. Hence, the fitness of $\mathrm{A}$ with the peering link is $P+f_{A}^{p}$ and the fitness of $\mathrm{B}$ is $-P+f_{B}^{p}$. The fitness of $\mathrm{A}$ and $\mathrm{B}$ without the peering link are $f_{A}^{n}$ and $f_{B}^{n}$, respectively. A's benefit due to the peering link is $P+f_{A}^{p}-f_{A}^{n}$, while that of $\mathrm{B}$ is $-P+f_{B}^{p}-f_{B}^{n}$. The fair price $P$ should equalize these benefits.

$$
\begin{aligned}
P+f_{A}^{p}-f_{A}^{n} & =-P+f_{B}^{p}-f_{B}^{n} \\
\text { i.e., } P & =\left(V_{B}-V_{A}\right) / 2
\end{aligned}
$$


The fair price is thus equal to half of the difference in the value of $\mathrm{A}$ and $\mathrm{B}$.

2) Optimality of peering with the fair price $P$ : In response to the fair price $P$ presented by the oracle, $\mathrm{A}$ and $\mathrm{B}$ each have two options: accept peering at price $P$ (meaning the peering link will be created) or deny peering. We determine whether accepting a peering link with the fair price given by equation (1) is the optimal strategy for A and B. This is modeled as a two-player game between A and B. The payoff matrix for this game is shown in table I.

\begin{tabular}{|c|c|c|}
\hline & Accept & Deny \\
\hline Accept & $P+f_{A}^{p},-P+f_{B}^{p}$ & $f_{A}^{n}, f_{B}^{n}$ \\
\hline Deny & $f_{A}^{n}, f_{B}^{n}$ & $f_{A}^{n}, f_{B}^{n}$ \\
\hline
\end{tabular}

TABLE I: Payoff matrix for the two-player game with price $P$.

The optimal strategies of $\mathrm{A}$ and $\mathrm{B}$ are best response strategies to the price $P$ offered by the oracle and the possible moves of the other network. Note that both the price $P$ and the possible moves of the other network ("accept" or "deny") are known. In the following, we determine the conditions under which peering is the optimal strategy for A and B using three commonly used definitions of optimal best-response behavior - maxi-min, expected payoff and minimum worst-case regret.

Maxi-min optimality:

In the maxi-min approach, network $\mathrm{A}$ attempts to maximize its minimum possible payoff, given the price $P$ and the possible decisions of network B. Thus, A maximizes its minimum payoff by accepting the peering link at price $P$ if

$$
\begin{aligned}
\min \left(P+f_{A}^{p}, f_{A}^{n}\right) & \geq f_{A}^{n} \\
\text { i.e., } P & \geq-V_{A}
\end{aligned}
$$

Similarly, B maximizes its minimum payoff by accepting the peering link at price $P$ if

$$
\begin{aligned}
\min \left(-P+f_{B}^{p}, f_{B}^{n}\right) & \geq f_{B}^{n} \\
\text { i.e., } P & \leq V_{B}
\end{aligned}
$$

Expected payoff optimality:

In this approach, network $\mathrm{A}$ attempts to maximize its expected payoff, given the compensation price $P$ and the possible decisions of B. If A accepts, its expected payoff is $\left(P+f_{A}^{p}+f_{A}^{n}\right) / 2$. If it denies, the expected payoff is $f_{A}^{n}$. The optimal strategy for A to maximize its expected payoff is to accept if

$$
\begin{aligned}
\left(P+f_{A}^{p}+f_{A}^{n}\right) / 2 & \geq f_{A}^{n} \\
\text { i.e. } \quad P & \geq-V_{A}
\end{aligned}
$$

and deny otherwise. Similarly, the optimal strategy for B is to accept if $P \leq V_{B}$, and deny otherwise.

Minimum worst-case regret optimality:

In this approach, a network attempts to minimize its worstcase "regret". Regret (or opportunity loss) is defined as the difference between the actual payoff and the maximum payoff that could have been obtained if a different course of action had been chosen. We compute the regret matrix of A by subtracting the maximum of each column from each entry of the payoff matrix in Table I. We compute the min-regret strategy for network A.

\begin{tabular}{|c|c|c|}
\hline & Accept & Deny \\
\hline Accept & 0 & 0 \\
\hline Deny & $f_{A}^{n}-f_{A}^{p}-P$ & 0 \\
\hline
\end{tabular}

TABLE II: Regret matrix for $\mathrm{A}$, in the case where $P+f_{A}^{p} \geq f_{A}^{n}$.

First, assume that $P+f_{A}^{p} \geq f_{A}^{n}$. A's regret matrix in this case is shown in Table II. To minimize regret, A accepts if

$$
0 \geq \min \left(f_{A}^{n}-f_{A}^{p}-P, 0\right)
$$

and denies otherwise, i.e., A accepts when $P \geq f_{A}^{n}-f_{A}^{p}$, and denies otherwise. Analysis of the case $P+f_{A}^{p}<f_{A}^{n}$ (omitted here due to space constraints) yields the same condition: the optimal min-regret strategy for $\mathrm{A}$ is to accept if $P \geq-f_{A}^{p}+f_{A}^{n}$, i.e., when $P \geq-V_{A}$ and deny otherwise. Symmetrically, B accepts if $P \leq f_{B}^{p}-f_{B}^{n}$, i.e., $P \leq V_{B}$ and denies otherwise.

Thus the optimal strategies for each of the three definitions of optimality are the same: A accepts if $P \geq-V_{A}$ and denies otherwise, and $\mathrm{B}$ accepts if $P \leq V_{B}$ and denies otherwise. The peering link will be created with the price $P$ proposed by the oracle, if "accept" is the optimal strategy for both A and $\mathrm{B}$ with that price. This implies that

$$
\begin{aligned}
P \geq f_{A}^{n}-f_{A}^{p} & \text { and } P \leq f_{B}^{p}-f_{B}^{n} \\
\left(V_{B}-V_{A}\right) / 2 \geq-V_{A} & \text { and }\left(V_{B}-V_{A}\right) / 2 \leq V_{B} \\
\text { i.e., } V_{A}+V_{B} & \geq 0
\end{aligned}
$$

As long as the aggregate value of the peering link for $A$ and $B$ is positive, forming a peering link according to the fair price $P$ is optimal for both $A$ and $B$. This implies that it may be optimal for $\mathrm{A}$ and $\mathrm{B}$ to form a peering link, even if the value of the link for one network is negative. The network with the negative value would be compensated by the other network via the price $P$.

3) Stability of a peering link with the fair price $P$ : We have shown that it is optimal for both $\mathrm{A}$ and $\mathrm{B}$ to accept the fair price $P$, as long as the aggregate value of the peering link is positive. We now study whether a peering link formed with the fair price $P$ is stable, i.e., do $\mathrm{A}$ and $\mathrm{B}$ have the incentive to unilaterally deviate and depeer the other network?

For a peering link with price $P$ to be stable, the (accept,accept) solution must be a Nash Equilibrium at the price $P$, i.e., no network should be able to get a better payoff by unilaterally deviating from the accept state. Using the payoff matrix in Table I,

$$
\begin{aligned}
& P+f_{A}^{p} \leq f_{A}^{n} \quad \text { and }-P+f_{B}^{p} \leq f_{B}^{n} \\
& \text { i.e. } \quad V_{A}+V_{B} \geq 0
\end{aligned}
$$

Which yields the same result as in equation (2). As long as the aggregate value of the peering link for $A$ and $B$ is positive, the peering link with price $P$ is stable. Using the payoff matrix in Table I, it is straightforward to see that as long as $V_{A}+V_{B}>0$, peering according to the fair price $P$ is a unique Nash Equilibrium. 


\section{$B$. Price setting by $A$ and $B$ individually}

The preceding discussion proposed a fair, optimal and stable price $P$ assuming a central oracle that knows the costs and fitness of $\mathrm{A}$ and $\mathrm{B}$. Next, we describe how $\mathrm{A}$ and $\mathrm{B}$ can individually determine the same compensation price as proposed by the oracle. We first assume that $\mathrm{A}$ and $\mathrm{B}$ have complete knowledge of their own value and the value of the other network. From the preceding analysis, paid peering is feasible only if the aggregate value of $\mathrm{A}$ and $\mathrm{B}$ is positive, i.e., $V_{A}+V_{B}>0$. In the following, we assume that this condition is satisfied.

We introduce the concept of "willingness to pay" of a network. Network A (B) determines the price it is willing to pay to $\mathrm{B}(\mathrm{A})$, and the price that it expects $\mathrm{B}(\mathrm{A})$ to pay, in order to achieve the fair compensation price $P$. Let $W_{A}$ and $W_{B}$ be the willingness to pay of $\mathrm{A}$ and $\mathrm{B}$, respectively. Let $R_{A}$ and $R_{B}$ be the payment that $\mathrm{A}$ and $\mathrm{B}$ respectively require from the other network.

A rational network A can use the following set of rules to determine the prices $W_{A}$ and $R_{A}$.

1) If $V_{A}<0$, then $W_{A}=0$ and $R_{A}=\frac{V_{B}-V_{A}}{2}$.

2) If $0<V_{A}<V_{B}$, then $W_{A}=0$ and $R_{A}=\frac{V_{B}-V_{A}}{2}$.

3) If $0<V_{B}<V_{A}$, then $W_{A}=\frac{V_{A}-V_{B}}{2}$ and $R_{A}=0$.

B evaluates a set of similar conditions to come up with the prices $W_{B}$ and $R_{B}$. Now, the peering link will be formed if

$$
W_{B} \geq R_{A} \text { and } W_{A} \geq R_{B}
$$

In the case of perfect knowledge, if $V_{B}>V_{A}$ then $W_{B}=$ $\frac{V_{B}-V_{A}}{2}$ and $R_{A}=\frac{V_{B}-V_{A}}{2}$. If $V_{B}<V_{A}$ then $R_{B}=\frac{V_{B}-V_{A}}{2}$ and $W_{A}=\frac{V_{B}-V_{A}}{2}$. The peering link will be formed, as $W_{B}=$ $R_{A}$ and $W_{A}=R_{B}$ in both cases. When $A$ and $B$ have perfect knowledge, they individually arrive at the same fair price as the centralized oracle.

\section{Price setting by $A$ and $B$ with uncertain value estimation}

In the previous section, we assumed that the total value of the peering link for $\mathrm{A}\left(V_{A}\right)$ and $\mathrm{B}\left(V_{B}\right)$ are accurately known to both parties. Here, we consider the case where A and B are not able to correctly estimate the value of the other network. Note that A and B are still able to accurately estimate their own value. We introduce the following notation to account for the estimated value terms. Let $V_{A}(B)$ denote B's estimate of A's value for the peering link and $V_{B}(A)$ denote A's estimate of B's value for the peering link.

As before, A calculates the quantities $W_{A}$ and $R_{A}$, while B calculates $W_{B}$ and $R_{B}$, but now using the estimated values $V_{A}(B)$ and $V_{B}(A)$. For $\mathrm{A}$ :

1) If $V_{A}<0$, then $W_{A}=0$ and $R_{A}=\frac{V_{B}(A)-V_{A}}{2}$.

2) If $0<V_{A}<V_{B}(A)$, then $W_{A}=0$ and $R_{A}=\frac{V_{B}(A)-V_{A}}{2}$.

3) If $0<V_{B}(A)<V_{A}$, then $W_{A}=\frac{V_{A}-V_{B}(A)}{2}$ and $R_{A}=0$.

B follows a similar procedure to determine $W_{B}$ and $R_{A}$. As before, the peering link is formed if

$$
W_{B} \geq R_{A} \text { and } W_{A} \geq R_{B}
$$

In the case of perfect estimation, $W_{B}$ and $R_{A}$ are always equal. This may not be the case when $\mathrm{A}$ and $\mathrm{B}$ have imperfect information. In that case, the eventual price can be any value in the range $\left[R_{A}, W_{B}\right]$. We study the various cases that could arise due to the estimation errors $V_{A}(B)-V_{A}$ and $V_{B}(A)-$ $V_{B}$. Without loss of generality, we consider a situation where $V_{A}<V_{B}$. With perfect knowledge, A requires a price $R_{A}=$ $\left(V_{B}-V_{A}\right) / 2$ and B is willing to pay $W_{B}=\left(V_{B}-V_{A}\right) / 2$.

\section{Value underestimation}

First, we assume that network $\mathrm{A}$ underestimates the value of the peering link for $\mathrm{B}\left(V_{B}(A)<V_{B}\right)$, while $\mathrm{B}$ estimates A's value correctly $\left(V_{A}(B)=V_{A}\right)$. This causes $R_{A}=$ $\frac{V_{B}(A)-V_{A}}{2}<\frac{V_{B}-V_{A}}{2}$ to decrease, meaning that A will accept a smaller price from B. $W_{B}=\frac{V_{B}-V_{A}(B)}{2}=\frac{V_{B}-V_{A}}{2}>R_{A}$, which means that the peering link is feasible, but $\mathrm{B}$ pays less than it would with perfect knowledge. If $\mathrm{B}$ underestimates $\left(V_{A}(B)<V_{A}\right)$, while A estimates correctly $\left(V_{B}(A)=V_{B}\right)$, then we find that the peering link is feasible, but $B$ will overpay. Thus, when one network underestimates the value of the peering link for the other, the network whose value is underestimated can benefit if it is able to correctly estimate the value of the other. It may thus be beneficial for a network to deliberately "hide"its value, causing the other network to underestimate it.

Next, consider that both $\mathrm{A}$ and $\mathrm{B}$ underestimate the value of the peering link for the other network $\left(V_{B}(A)<V_{B}\right.$ and $\left.V_{A}(B)<V_{A}\right)$. In this case, the willingness to pay of $\mathrm{B}$, $W_{B}=\frac{V_{B}-V_{A}(B)}{2}>\frac{V_{B}-V_{A}}{2}$ increases, while the requirement of $\mathrm{A}, R_{A}=\frac{V_{B}(A)-V_{A}}{2}<\frac{V_{B}-V_{A}}{2}$ decreases (or becomes 0 if $V_{B}(A)<V_{A}$, in which case $W_{A}>0$ ). This situation leads to the formation of the peering link, but both $\mathrm{A}$ and $\mathrm{B}$ can have a positive willingness to pay. The logical outcome here is for A and B to form a settlement-free peering link.

\section{The utility of hiding peering value}

The previous analysis showed that if network B underestimates the A's value for the peering link, then the peering link will be formed, but B pays more than the fair price. Assuming that A can estimate B's value correctly, A can benefit from hiding its true value. A still needs to ensure that the aggregate value from B's perspective is positive, i.e., $V_{A}(B)+V_{B}>0$, failing which the link is not formed as the fair price is no longer optimal and stable. Hence, A will attempt to make $V_{A}(B)$ appear as low as $-V_{B}$, but not lower. This, however, relies on the fact that $\mathrm{A}$ itself is able to accurately measure $V_{B}$. If this is not the case, then by hiding its own value, A runs the risk of making the peering link appear non-feasible to B.

\section{Value Overestimation}

First, we assume that network A overestimates the value of the peering link for $\mathrm{B}\left(V_{B}(A)>V_{B}\right)$, while B estimates A's value correctly $\left(V_{A}(B)=V_{A}\right)$. This causes $R_{A}=\frac{V_{B}(A)-V_{A}}{2}>$ $\frac{V_{B}-V_{A}}{2}$ to increase, meaning that $\mathrm{A}$ requires a larger price from B. However, $W_{B}=\frac{V_{B}-V_{A}(B)}{2}=\frac{V_{B}-V_{A}}{2}<R_{A}$. In this case, the peering link cannot be formed, as $W_{B}<R_{A}$. On the other hand, if B overestimates A's value, $\left(V_{A}(B)>V_{A}\right)$, while A estimates B's value correctly $\left(V_{B}(A)=V_{B}\right)$, then we have 
$W_{B}=\frac{V_{B}-V_{A}(B)}{2}<\frac{V_{B}-V_{A}}{2}$, meaning that B's willingness to pay decreases. $R_{A}=\frac{V_{B}^{2}(A)-V_{A}}{2}=\frac{V_{B}-V_{A}}{2}>W_{B}$. Again, the peering link cannot be formed, as $R_{A}>W_{B}$. Hence, in the case that one of the two networks overestimates the value of the other, the peering link cannot be formed.

Next consider that both $\mathrm{A}$ and $\mathrm{B}$ overestimate the value of the peering link for the other network $\left(V_{B}(A)>V_{B}\right.$ and $\left.V_{A}(B)>V_{A}\right)$. In this case, the willingness to pay of $\mathrm{B}$ decreases because $W_{B}=\frac{V_{B}-V_{A}(B)}{2}<\frac{V_{B}-V_{A}}{2}$, while the requirement of $\mathrm{A}$ increases as $R_{A}=\frac{V_{B}(A)-V_{A}}{2}>\frac{V_{B}-V_{A}}{2}$. The peering link will not be formed. Value overestimation is common in the real world, where one or both networks believe that the other sees a larger value for the peering link. The peering link is not created, when it would actually benefit both networks to do so.

Note that in this work, we have not considered the possibility that the two networks engage in negotiations in case their willingness to pay and payment requirements are incompatible. In case the networks negotiate and reveal their true value for the peering link, the situation reduces to the case of perfect information, in which case equation 4 leads to the formation of a fair and stable peering relationship.

\section{Practical issues: MeAsuring VAlues}

In this section, we discuss the practical issues faced by networks $\mathrm{A}$ and $\mathrm{B}$ in measuring the values $V_{A}, V_{B}(A), V_{B}$ and $V_{A}(B)$. Let $\mathcal{C}_{A}$ and $\mathcal{C}_{B}$ be the set of networks in the customer tree of $\mathrm{A}$ and $\mathrm{B}$, respectively. Let $L_{A}^{p}, T_{A}^{p}$ and $R_{A}^{p}$ be the local cost, transit cost, and revenue of $\mathrm{A}$ when the peering link is present, and $L_{A}^{n}, T_{A}^{n}$ and $R_{A}^{n}$ be the corresponding values when the peering link is not present. The fitness of A with and without the link is given by $f_{A}^{p}=R_{A}^{p}-L_{A}^{p}-T_{A}^{p}$ and $f_{A}^{n}=R_{A}^{n}-L_{A}^{n}-T_{A}^{n}$. A's value from the peering link is

$V_{A}=f_{A}^{p}-f_{A}^{n}=\left(R_{A}^{p}-R_{A}^{n}\right)-\left(L_{A}^{p}-L_{A}^{n}\right)-\left(T_{A}^{p}-T_{A}^{n}\right)$

To measure $V_{A}$, network A must measure the change in its revenue, local costs and transit costs due to the peering link. Similarly, to estimate $V_{B}(A)$, A must estimate the changes in these quantities for for ISP $B$.

\section{Traffic fluctuations}

By creating a new peering link, A and B divert traffic that is currently exchanged through an intermediate transit provider to the newly established peering link. This diversion of traffic from transit links to the new peering link affects the transit costs of $A$ and $B$. In addition, $A$ and $B$ can attract traffic by providing shorter paths between networks in $\mathcal{C}_{A}$ and $\mathcal{C}_{B}$. They can also lose traffic, as customers may no longer route their traffic through them (if the peering link leads to a longer path or due to local policies enforced by those customers). As A and B get paid by their customers based on the traffic volume they transit, attraction and loss of traffic affects the revenues of $A$ and $B$. Finally, A and B could see internal route changes, i.e., change in the ingress or egress point of existing traffic. Internal route changes affect traffic flow in networks $A$ and $B$, and affect the internal costs of $A$ and $B$.

\section{Peering trial}

A practical method to measure traffic fluctuations and the effects on the values of $\mathrm{A}$ and $\mathrm{B}$ is to set up the peering link for a "trial" period. Such trials are commonly used by networks present at Internet Exchange Points (IXPs), to check whether a potential peer meets peering criteria. Using routing and Netflow data collected before and during the trial period, A has sufficient information to measure its own value $V_{A}$, as well as make an estimate of the value $V_{B}(A)$ for $\mathrm{B}$. Note that monitoring overhead during the peering trial is low, and can be continued after the trial period. A network can thus also detect a peer that cheats by sending a different traffic profile during the peering trial.

\section{A. Finding its own value for the peering link}

It is straightforward for A to measure the amount of traffic that shifts from transit providers to the new peering link, and the resulting change in transit cost $T_{A}^{p}-T_{A}^{n}$. ISP A can use Netflow data collected before and during the peering trial to measure the set of source-destination pairs $(s, d)$ for which traffic traverses the peering link. If traffic for a $(s, d)$ pair traversed A before the peering trial, but no longer traverses A during the trial, then this is an indication that $\mathrm{A}$ lost the traffic for $(s, d)$. A similar approach gives the amount of new traffic that A attracts. ISP A uses the measured values of traffic attracted and lost to determine the change in its revenue $R_{A}^{p}-$ $R_{A}^{n}$. To measure internal routing variation, A compares the ingress and egress points of A's traffic with and without the peering link. Using information about A's topology and local cost structure, A can measure the change in local cost $L_{A}^{p}-L_{A}^{n}$. Network A can thus measure all three components of its value function in equation (6), and can accurately measure $V_{A}$.

\section{B. Evaluating the potential peer's value for the peering link}

Network B's cost and revenue structure is critical information that it is not likely to share, especially with potential peers. Network A mus approximate the B's value for the peering link.

To estimate the change in B's revenue $\left(R_{B}^{p}-R_{B}^{n}\right)$ due to the peering link, A must estimate the amount of traffic B attracts or loses. Consider a source-destination pair $(s, d)$ that ingresses A through the peering link. ISP A must estimate whether $(s, d)$ is traffic attracted by B. This is non-trivial, as A must guess how $(s, d)$ was being routed (in particular, if the route traversed B) before and during the peering trial. Though techniques have been proposed to predict AS paths using public BGP data [2], A will not be able to accurately measure B's change in revenue. Due to difficulties in measuring B's internal topology and costs, A can only approximate B's change local costs $\left(L_{B}^{p}-L_{B}^{n}\right)$. A can oversimplify by assuming that B's revenue and internal costs do not change.

To measure B's change in transit costs $\left(T_{B}^{p}-T_{B}^{n}\right)$, A needs to measure the amount of traffic that $B$ shifts from transit providers to the peering link. For this purpose, network A measures the total traffic $T_{B A}$ that it receives over the peering link, and assumes that all this traffic was initially being routed through B's providers. Next, A finds B's existing 
transit providers using public BGP data. A also finds the pricing parameters of B's providers, either directly from those providers, or from transit pricing surveys [3]. Due to the concavity of transit pricing, it is difficult for A to exactly calculate the transit savings for $\mathrm{B}$, due to the peering link. To do so, A needs to know the amount of traffic B sent to its providers before the peering trial. By estimating a ballpark figure for B's total transit traffic, A can approximate the transit savings of $\mathrm{B}\left(T_{B}^{p}-T_{B}^{n}\right)$. Thus, with approximations of the terms in equation 6, ISP A can estimate $V_{B}(A)$.

In summary, ISP A can accurately measure its own value $V_{A}$, but A's estimation of B's value $\left(V_{B}(A)\right)$ is necessarily an approximation due to 1) Inaccuracies in measuring traffic fluctuations for B. 2) Inaccuracies in measuring B's internal costs. 3) Inaccuracies in measuring B's transit savings. We study the effect of inaccurate value estimation on the resulting peering links in Section V.

\section{The Global EFFECTS OF VALUE-BASED PEERING}

We use the ITER [1] framework to evaluate the impact of value-based peering on the global Internet. ITER is a first-principles model of interdomain network formation that incorporates the effects of economics, traffic flow, geography, pricing/cost structures and interdomain routing policies. ITER uses an agent-based computational method to find the equilibrium that results as each network uses a certain provider and peer selection strategy. ITER models the following types of networks: Enterprise Customers (EC) are stub networks at the periphery of the Internet. ECs do not peer and do not have customers; their only action is provider selection. Content providers $(\mathrm{CP})$ are also stub networks (no customers) that differ from ECs in two ways. First, they are major sources of traffic, and second, they can also form peering links. Small Transit Providers (STP) and Large Transit Providers (LTP) are ANs whose main business function is to provide Internet connectivity to their transit customers (other ANs). STPs are transit providers with limited geographical presence (regional scope), while LTPs are transit providers with almost global presence. In ITER, we model two different peering criteria that networks use to select peers. In the traffic ratio criteria (TR), a network $i$ agrees to peer with a network $j$ if the traffic $T_{i j}$ that $i$ sends to $j$ is approximately equal to the traffic $T_{j i}$ that $j$ sends to $i$ (the traffic ratio $T_{j i} / T_{i j}<t_{i}$, where $t_{i}$ is $i$ 's traffic ratio threshold). In peering by cost-benefit analysis (CB), two networks agree to peer if both networks benefit from creating the peering link. Networks use a price-based (PR) method (simply choose the cheapest providers) or pricebased selective (SEL) method (choose the cheapest providers that are not STPs) to choose their upstream providers.

In ITER, networks A and B peer using a "peering trial", i.e., they initially create a peering link and determine respectively their resulting fitness. If the peering link results in higher fitness for both $A$ and $B$, then the peering link is retained. Each network periodically re-evaluates unilaterally its set of peers using a "depeering trial". A network (say A) temporarily removes its peering link with network $\mathrm{B}$, and evaluates its resulting fitness. If the fitness of $A$ is better without the peering link, then A unilaterally depeers $B$, otherwise the peering link is retained. We implement the value-based peering model in ITER as follows. Using a peering trial for two candidate networks $\mathrm{A}$ and $\mathrm{B}$, we calculate $V_{A}$ and $V_{B}$, i.e., the actual value of the peering link for $\mathrm{A}$ and $\mathrm{B}$. We then simulate imperfect estimation by distorting these values to get $V_{A}(B)$ (the value of $\mathrm{A}$ as estimated by $\mathrm{B}$ ) and $V_{B}(A)$ (the value of $\mathrm{B}$ as estimated by A). $V_{A}(B)=V_{A}+e_{A} *\left|V_{A}\right|$ and $V_{B}(A)=V_{B}+e_{B} *\left|V_{B}\right|$. where $e_{A}$ and $e_{B}$ represent the errors in estimating the value of $\mathrm{A}$ and $\mathrm{B}$, respectively. If $e_{A}$ is positive, A's value is overestimated, while if $e_{A}$ is negative, A's value is underestimated. A and B now calculate $W_{A}$ and $W_{B}$, their willingness to pay, and $R_{A}$ and $R_{B}$, their "requirement" from the link, as per the rules in Section III-B. If $W_{A}>=R_{B}$ and $W_{B}>=R_{A}$ the peering link is formed, and the price $P$ is set to $W_{B}-W_{A}$.

We define the following baseline parameterization of ITER:

1. Edge networks (ECs and CPs) use price based (PR) provider selection. STPs and LTPs use price based selective (SEL) provider selection.

2. We consider two types of peering for CPs, STPs and LTPs: cost-benefit (CB) or traffic ratio (TR) peering.

3. The interdomain traffic matrix is mainly client-server in nature, with large traffic volumes from CPs to ECs.

4. Transit and peering prices show economies of scale. We choose parameters from Norton's transit cost survey [3].

5. Due to its computational complexity, ITER has to be run at a small scale, with a total of 210 networks -4 LTPs, 16 STPs, $10 \mathrm{CPs}$, and 180 ECs.

The paid-peering scenario is the case where STPs, LTPs and CPs engage in paid-peering relationships using the valuebased peering model. We use the following ranges for the $e$ values of each network: $\{[-0.25,0.25]\},\{[-0.5,-0.25] \cup$ $[0.25,0.5]\},\{[-1,-0.5] \cup[0.5,1]\},\{[-1.5,-1] \cup[1,1.5]\}$. For each ITER scenario, we report output metrics averaged across 50 simulation runs.

The density of peering links. We compare the density of peering links (as a fraction of all links) with different ITER scenarios, as shown in Table III (The numbers following " \pm " denote the standard deviation across 50 simulation runs). We first present results where $e$ is drawn from two-sided ranges. We find that the value-based peering model without uncertainty results in the highest fraction of peering links at equilibrium. It is intuitive that value-based peering with $e=0$ results in more peering links than traffic-ratio or cost-benefit based peering. In the case of cost-benefit peering, a peering link is established only if $V_{A}$ and $V_{B}$ are both positive, i.e., both $A$ and $B$ see increased fitness with the peering link. With value-based peering, however, the peering link is formed if $V_{A}+V_{B}>0$, even if one of $V_{A}$ or $V_{B}$ is negative. We find that as the error in value estimation increases, the number of peering links at equilibrium decreases.

We also simulated the scenario where $e$ is one-sided, drawn from the ranges $[-2,-1.5],[-1.5,-1],[-0.5,0],[0.5,1],[1.5,2]$. When $e<0$, all networks underestimate the value of a 


\begin{tabular}{|c|c|}
\hline scenario & fraction of peering links \\
\hline TR & $2.9 \pm 0.1$ \\
CB & $7.0 \pm 0.23$ \\
VB, $=0$ & $10.5 \pm 0.32$ \\
VB, $\in \in[-0.25,0.25]$ & $7.15 \pm 0.68$ \\
VB, $\mathrm{e} \in \pm[0.25,0.5]$ & $7.08 \pm 0.58$ \\
VB, $\mathrm{e} \in \pm[0.5,1]$ & $6.88 \pm 0.44$ \\
VB, $\mathrm{e} \in \pm[1,1.5]$ & $6.19 \pm 0.37$ \\
$\mathrm{VB}, \mathrm{e} \in \pm[1.5,2]$ & $5.05 \pm 0.33$ \\
\hline
\end{tabular}

TABLE III: The fraction of peering links at equilibrium. e $\in \pm[0.25,0.5]$ denotes a two-sided error in the range $[-0.25,-0.5] \cup[0.25,0.5]$.

peering link, while when $e>0$, all networks overestimate the value. We find that when $e<0$, the fraction of peering links is larger than when $e=0$. When $e>0$ the fraction of value-based peering links drops almost to zero, and the only peering links are between LTPs. This confirms our results in section III-C, which state that overestimation of value by one or both networks precludes the formation of peering links, while underestimation allows more peering links.

We compare the average end-to-end path length (in terms of network hops) in the equilibrium network under different scenarios. We find that the increased density of peering links with value-based peering results in shorter average end to end paths. The average path length with value-based peering is 3.7, while it is 4.0 with traffic ratio or cost-benefit peering.

Which networks are involved in paid-peering links? Next, we classify peering links in the equilibrium network according to the type of network they connect, shown in Table IV. We find that links between LTPs and CPs cannot be formed with traffic-ratio peering (because the traffic ratio between LTPs and CPs can never be balanced). Such links are formed with cost-benefit peering, as well as with valuebased peering. Further, the number of links between LTPs and CPs is relatively insensitive to $e$. On the other hand, peering links between CPs and STPs are not formed with cost-benefit peering. This is because in this scenario, CPs are able to form peering links with LTPs ${ }^{2}$. Once a CP peers with LTPs at the top of the hierarchy, it can reach most destinations through those those peering links. Consequently, a CP sees negative value for a link with an STP, while the STP sees positive value. The CP-STP link cannot be formed with cost-benefit peering, as both parties must see positive value. With value-based peering, the CP-STP peering link can be formed, because the aggregate value of the peering link is positive. In such cases, the STP pays the CP. The number of CP-STP peering links is sensitive to $e$, however, and decreases quickly as $e$ is increased.

\begin{tabular}{|c|c|c|c|c|}
\hline scenario & STP-STP & STP-LTP & STP-CP & LTP-CP \\
\hline TR & $3.0 \pm 0$ & $0.2 \pm 0.3$ & $0 \pm 0$ & $0 \pm 0$ \\
CB & $3.9 \pm 0.3$ & $2.2 \pm 0.4$ & $0 \pm 0$ & $10.3 \pm 0.5$ \\
VB, e=0 & $4.0 \pm 0$ & $2.4 \pm 0.8$ & $11.7 \pm 0.8$ & $10.3 \pm 0.7$ \\
$\mathrm{VB}, \mathrm{e} \in[-0.25,0.25]$ & $4.4 \pm 0.5$ & $1.0 \pm 1$ & $1.7 \pm 1.2$ & $9.5 \pm 0.7$ \\
$\mathrm{VB}, \mathrm{e} \in \pm[0.25,0.5]$ & $3.6 \pm 0.8$ & $1.4 \pm 1$ & $1.6 \pm 1.3$ & $9.7 \pm 1$ \\
$\mathrm{VB}, \mathrm{e} \in \pm[0.5,1]$ & $3.4 \pm 0.9$ & $1.4 \pm 0.8$ & $1.1 \pm 1$ & $9.6 \pm 1$ \\
$\mathrm{VB}, \mathrm{e} \in \pm[1,1.5]$ & $3.0 \pm 0.5$ & $0.8 \pm 0.7$ & $0.2 \pm 0.3$ & $9.3 \pm 0.7$ \\
\hline
\end{tabular}

TABLE IV: The number of peering links between various network types

Which networks pay with value-based peering? We de-

\footnotetext{
${ }^{2}$ Cost-benefit peering between LTPs and CPs is further discussed in [1].
}

termine the direction of payment for each value-based peering link in the equilibrium network. Table $\mathrm{V}$ shows the number of value-based peering links in which a certain network type pays another, and the standard deviation across 50 simulation runs. The most interesting case is with value-based peering and $e=0$. In that case, we find a significant number of links in which an STP pays a CP, and a comparable number of links where a $\mathrm{CP}$ pays an LTP. This means that the same type of network can pay in some value-based peering relationships, while it can get paid in others.

CPs pay certain networks, and get paid by others, because of the following reason. With value-based and cost-benefit peering, CPs are able to peer with LTPs. In the cost-benefit model, peering is settlement-free, and neither network pays the other. With value-based peering, CPs can peer with LTPs at the top of the hierarchy, but CPs must pay LTPs; the value of the peering link is much higher for CPs than for LTPs. Once such CP-LTP links are established, CPs can reach all networks in the customer tree of those LTPs. Consequently, the $\mathrm{CP}$ sees little value in peering with an STP lower in the hierarchy. This makes the value of a CP-STP link negative for the CP, while this value is positive for the STP. For STPs and CPs to peer, STPs must pay the CPs. We thus see links where STPs pay CPs, and also links where CPs pay LTPs.

\begin{tabular}{|c|c|c|c|c|c|}
\hline VB scenario & STP $\rightarrow$ LTP & STP $\rightarrow$ CP & LTP $\rightarrow$ STP & LTP $\rightarrow$ CP & CP $\rightarrow$ LTP \\
\hline $\mathrm{e}=0$ & $1.2 \pm 1$ & $11.7 \pm 0.9$ & $1.2 \pm 0.9$ & $0.6 \pm 0.7$ & $9.8 \pm 0.5$ \\
$\mathrm{e} \in[-0.25,0.25]$ & $0.5 \pm 0.7$ & $1.7 \pm 1.2$ & $0.5 \pm 0.7$ & $1.1 \pm 0.7$ & $8.4 \pm 0.7$ \\
$\mathrm{e} \in \pm[0.25,0.5]$ & $1.1 \pm 0.5$ & $1.6 \pm 1.3$ & $0.3 \pm 0.7$ & $1.2 \pm 0.9$ & $8.5 \pm 0.9$ \\
$\mathrm{e} \in \pm[0.5,1]$ & $1.1 \pm 0.5$ & $1.1 \pm 1$ & $0.3 \pm 0.7$ & $1.1 \pm 0.9$ & $8.5 \pm 0.9$ \\
$\mathrm{e} \in \pm[1,1.5]$ & $0.7 \pm 0.6$ & $0.2 \pm 0.3$ & $0.1 \pm 0.3$ & $1.8 \pm 1.1$ & $7.5 \pm 1.2$ \\
\hline
\end{tabular}

TABLE $V$ : The direction of payment in value-based peering links

How does value-based peering affect the fitness of different network types? We study how value-based peering affects the fitness of different network types. Table VI shows the average fitness of STPs, LTPs and $\mathrm{CPs}^{3}$ under various types of peering. We find that the change from TR to CBpeering results in lower fitness for STPs. With CB-peering, CPs can peer with LTPs at the top of the hierarchy and reach most networks via those peering links; CPs then do not need to choose STPs as providers. Further, CPs see no value in settlement-free peering with STPs, due to which STP fitness decreases. We find that with value-based peering, STPs can peer with CPs by paying them the fair price, which is better for STPs than not peering at all. Even though STPs pay CPs in these paid-peering links, STPs see better overall fitness with paid-peering as compared to CB-peering. This benefit for STPs, however, declines with increasing uncertainty in the peering price evaluation, mainly because the number of STP-CP links decreases. We also find that the fitness of LTPs increases as $e$ is increased. This is because with a larger error, more CP-LTP links are formed, and CPs pay more than the fair price for those links.

\footnotetext{
${ }^{3}$ The fitness of CPs is mostly negative, because ITER does not model their revenue sources.
} 


\begin{tabular}{|c|c|c|c|}
\hline scenario & STP fitness (\$k) & LTP fitness (\$k) & CP fitness (\$k) \\
\hline TR & $52 \pm 0$ & $44 \pm 0$ & $-82 \pm 0$ \\
CB & $27 \pm 1.8$ & $61 \pm 9$ & $-59 \pm 3.5$ \\
VB, e=0 & $31 \pm 2.2$ & $42 \pm 9$ & $-59 \pm 3.8$ \\
VB, e $\in[-0.25,0.25]$ & $26 \pm 3.9$ & $113 \pm 18$ & $-79 \pm 3.6$ \\
$\mathrm{VB}, \mathrm{e} \in \pm[0.25,0.5]$ & $26 \pm 3.4$ & $104 \pm 14$ & $-76 \pm 4.5$ \\
$\mathrm{VB}, \mathrm{e} \in \pm[0.5,1]$ & $24 \pm 3.5$ & $104 \pm 15$ & $-74 \pm 5.4$ \\
$\mathrm{VB}, \mathrm{e} \in \pm[1,1.5]$ & $21 \pm 2.5$ & $107 \pm 15$ & $-71 \pm 5.2$ \\
\hline
\end{tabular}

TABLE VI: The average fitness of different network types

\section{RELATED WORK}

There has been some recent work on studying ISP interconnection using non-cooperative game theory [4]-[8]. These models vary in complexity, dealing with issues such as asymmetric networks, hot and cold potato routing, and different cost structures. This work mainly investigated the conditions under which networks can benefit from settlement-free peering. A series of papers [9]-[13] study multi-player network formation, where Autonomous Systems form a graph to route traffic between themselves. Our work differs from these previous approaches in that we study bilateral contracts between two networks, allowing for paid-peering. We highlight the practical difficulties faced by networks in estimating the value of a peering link. We also consider the effects of inaccurate value estimation on the formation of peering links. We implemented value-based peering in an agent-based model parameterized with realistic pricing/cost data, business types of ASes, and interdomain traffic matrices. This allows us to study the effects of value-based peering on topological properties of the global Internet (e.g., path lengths), profitability of different network types, and the direction of payment on paid-peering links.

Recent work attempts to model the evolution of the Internet topology, studying provider and peer selection methods and their effects on the global Internet [1], [14]. These papers focus mainly on settlement-free peering, and are more concerned with the global impact of relatively simple peer and provider selection methods. We use the ITER model from [1] to evaluate the global effects of value-based peering.

Norton discusses how economic and competitive interests influence peering and transit connectivity [15]. Norton also discusses the "peering playbook" [16], a practical guide for ISPs to choose between peering and transit connectivity. The "peering simulation game" [17] enacts the negotiations in peering relationships, and concludes that randomness and the personalities of the involved parties play a crucial role. Norton's work gives us insights into how settlement-free interconnection decisions are made in practice. It lacks, however, a theoretical framework that can be used to study the rationality of various policies. Finally, from a policy standpoint, Farratin et al. [18] discuss the emergence of paid peering, highlight the increased complexity of the negotiations involved, and discuss the implications on telecommunications policy.

\section{CONCLUSIONS}

We proposed a quantitative, value-based framework for Internet peering agreements. We derived a paid-peering price that is fair (both networks see equal benefit at that price), optimal (peering at that price is the best strategy for both networks) and stable (no network has the incentive to unilaterally depeer the other network). We showed that the two networks can individually arrive at the optimal price, if they can accurately measure the value of the link. We also considered the case where a network is not able to accurately measure the value of the peering link for the other network. We showed that overestimation of the peering value precludes the formation of peering links that should otherwise have been formed. On the other hand, a network can benefit from "hiding" its own value (causing the other network to underestimate it), under the condition that it is able to estimate the other's value correctly. Finally, we studied the global effects of value-based peering. We found that value-based peering leads to a higher density of peering links, and shorter end-to-end paths. Interestingly, we found that content providers can be on both sides of a paid peering relationship, as they pay large transit providers, but get paid by smaller regional providers.

\section{REFERENCES}

[1] A. Dhamdhere and C. Dovrolis, "A Model of Interdomain Network Formation, Economics and Routing," Georgia Tech Technical Report GT-CS-09-09, 2009.

[2] W. Muhlbauer, A. Feldmann, O. Mannel, M. Roughan, and S. Uhlig, "Building an AS-Topology Model that Captures Route Diversity," in Proc. ACM SIGCOMM, Pisa, Italy, 2006.

[3] W. B. Norton, "Transit Cost Survey," www.nanog.org/mtg-0606/pdf/bill. norton.2.pdf, Jul 2006.

[4] E. Jahn and J. Prfer, "Interconnection and competition among asymmetric networks in the Internet backbone market," Information Economics and Policy, Elsevier, 2007.

[5] N. Badasyan and S. Chakrabarti, "A simple game-theoretic analysis of peering and transit contracting among internet service providers," Telecommunications Policy, vol. 32, no. 1, 2008.

[6] S. Lippert and G. Spagnolo, "Internet peering as a network of relations," Telecommunications Policy, 2008.

[7] G. Shrimali and S. Kumar, "Paid peering among internet service providers," in Proc. GameNets workshop on Game theory for communications and networks, 2006.

[8] L. He and J. Walrand, "Pricing and Revenue Sharing Strategies for Internet Service Providers," IEEE Journal on Selected Areas in Communications, May 2006.

[9] R. T. Ma, D. Chiu, J. C. Lui, V. Misra, and D. Rubenstein, "On Cooperative Settlement Between Content, Transit and Eyeball Internet Service Providers," in Proc. ACM CoNEXT, 2008.

[10] R. T. B. Ma, D. Chiu, J. C. S. Lui, V. Misra, and D. Rubenstein, "Internet Economics: the use of Shapley Value for ISP Settlement," in Proc. ACM CoNEXT, 2007.

[11] E. Anshelevich, A. Dasgupta, E. Tardos, and T. Wexler, "Near-optimal Network Design with Selfish Agents," in Proc. STOC, 2003.

[12] E. Anshelevich, B. Shepherd, and G. Wilfong, "Strategic Network Formation through Peering and Service Agreements," in Proc. FOCS, 2006.

[13] R. Johari, S. Mannor, and J. Tsitsiklis, "A Contract-based Model for Directed Network Formation," Games and Economic Behavior, vol. 56, no. 2, August 2006.

[14] H. Chang, S. Jamin, and W. Willinger, "To Peer or Not to Peer: Modeling the Evolution of the Internet's AS-Level Topology," in Proceedings of IEEE Infocom, 2006.

[15] W. B. Norton, "A Business Case for ISP Peering," Equinix white papers, 2002.

[16] - , "The Art of Peering: The Peering Playbook," Equinix white papers, 2002.

[17] — "The Peering Simulation Game," Equinix white papers, 2002.

[18] P. Faratin, D. Clark, P. Gilmore, S. Bauer, A. Berger, and W. Lehr, "Complexity of internet interconnections: Technology, incentives and implications for policy," in 35th Annual Telecommunications Policy Research Conference, 2007. 